\title{
ABSTRACT \\ CONJUNCTIVE AND DISJUNCTIVE THINKING IN CHILDREN
}

\author{
Catherine E. Snow
}

In Iight of the finding (Bruner, 1956) that disjunctive concepts are more difficult for adults than conjunctive concepts, a developmental study of the relative difficulty of conjunctive and disjunctive concepts was undertaken. Five groups of children, ranging in age from five years to thirteen years, were tested on logically equivalent conjunctive and disjunctive concepts. It was found that children of all ages make more errors on disjunctive than on conjunctive tasks. The strategies used for conjunctive tasks were more appropriate at all age levels. Because of the fact that the difference in difficulty is quite constant over the age groups, it was concluded that some aspect of conjunctive groupings is more natural. As a result, they occur more frequently and there is greater opportunity to learn to reason correctly about them. 
CONJUNCTIVE AND DISJUNCTIVE THINKINE IN CHILDREN

by

\title{
CATHERINE SNOW
}

\begin{abstract}
A thesis submitted to the Faculty of Graduate Studies and Research in partial fulfillment of the requirements for the degree Master of. Arts.
\end{abstract}

Department of Psychology

McGill University

Montreal

August, 1967

(c) Catherine Snow 1968 
ACKNOWLEDGEMENTS

I should like to express sincere thanks to Mr. Robert Leicester, principal of St. George's School, and his staff for the kindness with which they offered their facilities and assistance. I should also like to thank Mr. Andrew MacDonald and the staff of the Westmount YMCA for their help. 
TABLE OF CONTENTS

\begin{tabular}{lr} 
INTRODUCTION & $\begin{array}{c}\text { Page } \\
\text { METHOD }\end{array}$ \\
RESULTS & 6 \\
DISCUSSION & 12 \\
SUMMARY & 17 \\
REFERENCES & 23 \\
\hline
\end{tabular}


INTRODUCT ION

In the book A Study of Thinking by Bruner, Goodnow, and Austin (1956) it was first noted that adults find working with disjunctive concepts very difficult. Intelligent adults normally choose efficient and logically justifiable strategies when faced with the task of finding the correct conjunctive cancept. However, these same adults become quite inefficient and confused when presented with an analogous task in which the concept is disjunctive. Typically, they attempt to use the strategies which were correct and efficient for conjunctive concepts, even though they are now faced with a different task where these conjunctive strategies are no longer effective. It was suggested by Bruner (1956) that the source of the difficulty with disjunctive concepts lay in the common preference for positive tests of hypotheses. This preference was first pointed out by Hovland and Weiss (1953) in an experiment dealing with conjunctive concepts. No experimental studies have been done in an attempt to understand the basis of the preference for positive tests, or the possible relationship of this preference to the greater ease of conjunctive thinking. Nor have any further studies of disjunctive reasoning been done.

Conjunction is normally expressed by the word "and". In terms of symbolic logic, a conjunction refers to a proposition which is true only if all of its terms are true. In terms of the tasks typically used to study concept formation, a conjunction is a concept 
having two terms, where positive instances all represent both terms of the concept. Disjunction, normally expressed by the word "or", refers to a logical proposition which is true if any of its terms is true. A disjunctive concept, then, is one having two or more terms, where positive instances display any one of the terms, not necessarily all. There is one major difference between conjunctive and disjunctive concepts which should be noted. This is the fact that information about conjunctive concepts can be gained most efficiently from positive instances of the concept, whereas negative instances give more information about disjunctive concepts. This is true because positive instances of disjunctive concepts may represent either term of the concept, or both terms, and there is no way of knowing, which is the case. Negative instances, however represent neither term, and therefore can give information about both of them.

Adults, then, seem to have a bias toward conjunctive thinking. In a logical sense, disjunctive concepts are no more difficult than conjunctive, if the same number of possible hypotheses is present in both cases. Although the logical difficulty is the same, the psychological difficulty is not. It is possible that this is so because the human brain is somehow programmed for conjunctive thinking. Or it is possible that the difference arises as a result of experience in a world and a language system where conjunction is more common. Bruner (1956) presents much common sense evidence in support of the idea that our environment promotes conjunctive rather than disjunctive thought. He states, for instance, that the basis of scientific reasoning is the assumption that common effects have common causes, which disjunctive 
categorizing violates. In addition, we use a language system where a conjunctive category is created every time a noun is modified "green apples" refers to the class of all things which are both apples and green) but where disjunctive categories require rather more clumsy verbal labèls.

Is it then true that conjunction is easier because we have had more experience with it? One way of approaching this question is through a developmental study. Presamably if a child learns conjunctive reasoning and has much less opportunity to learn disjunctive reasoning; then in very young children who have had the least oppartunity for cultural learning, there should be little difference between the difficulty of conjunctive and disjunctive tasks, or at least less difference than for older children. If the greater difficulty of disjunctive reasoning is largely environmentally induced, then one would predict developmental curves for the two kinds of tasks which diverge as age increases. On the other hand, if disjunctive reasoning is inherently more difficult, then one would expect parallel curves.

It is, of course, impossible to obtain a complete developmental curve; children have to have a certain level of verbal and conceptual development before they are able to understand a concept formation task. But in order for such a study to have any value, children as young as five years must be tested, because by the time they start school the cultural influences have become very strong. The problem is to choose a task simple enough for five-year-olds, yet difficult enough so that the same task could be given to children as old as thirteen. It seemed also that further information could be gained if the task were the same 
in kind as that used by: Bruner with adults, though of course not so difficult. The extensive studies done on concept formation in children by Bruner, Olver, Greenfield, et al., (1966), reported in Studies in Cognitive Growth, generally use tasks quite different from those used with adults. In addition, recognizing the fact that there are probably qualitative changes in children's thinking as they grow older, the task should be one which is as comprehensible to a child in any of the three stages identified by Piaget (1954) and by Bruner (1966). Since children at all three age levels are to be tested, the task must be capable of a solution at a perceptual level, and must not be designed strictly in terms of abstract solutions.

Only slight modifications of the tasks used by Bruner (1956) with adults are necessary to achieve these ends. Concept cards representing several atiributes, each attribute having two or three values, can be used with children if the attributes are all perceptually obvious and if there are not too many of them. So as to make the task possible for children still relying on perceptual modes of thought, and to eliminate a memory factor, all the information already accumulated should be available. This can be achieved simply by allowing the subject to look at all the cards which have already been identified as positive or negative instances. Sorting cards into two piles, where the aim is to place cards correctly, is a much more concrete task than trying to attain a concept as quickly as possible, and requires considerably less abstraction, but is as good a reflection of the thought processes. A task essentially the same as Bruner's could be chosen with only these two changes; all the cards are available to the subject at 
all times, and his primary aim is to sort cards correctly, stating the concept being a secondary aim:

A further value of this kind of developmental study is to examine the differences in the strategies used by adults and children, and to note at what ages different strategies first appear. A task in which a child sorts cards reveals "selection strategies", or the at-tempts to increase information and avoid error by choosing cards in one order rather than another. Of specific interest is the question of whether children, like adults, use strategies appropriate for conjunctive tasks with all tasks, or use different strategies for the different kinds of tasks, or fail to use conjunctive strategies at all. 
METHOD

Subjects

The subjects were 97 children divided into five age groups as follows:

I. 5 years, 6 months to 6 years, 4 months: 10 boys, 10 girls

2. 7 years, 0 months to 8 years, 2 months: 10 boys, 10 girls

3. 8 years, 5 months to 9 years, 5 months: 10 boys, 10 girls

4. 10 years, 5 months to 11 years, 7 months: 10 boys, 10 girls

5. 12 years, 2 months to 13 years, 11 months: 10 boys, 7 girls

The children in the secand and fifth groups were selected from among participants in a Montreal YMCA summer program. The other subjects were students at a Montreal independent school. This is a private school which draws students from high-income, high-intelligence families. Students to be tested were selected by their teachers, who had been instructed to choose "average" students. On the basis of classes for which intelligence scores were available, the average student is of somewhat above average I.Q. Subjects were selected from the YMCA summer program in an attempt to match the background, educational level, and intelligence of the independent school students.

\section{Materials}

Four decks of concept cards were used. One pair of decks, deck $1-a$ and deck 1-b, was selected from the set of cards having the following attributes and values. 
Attribute

card color
figure color
shape of figure
size of figure
number of figures

\section{Values}

$$
\begin{aligned}
& \text { yellow or white. } \\
& \text { red or blue } \\
& \text { square or circle } \\
& \text { large or small } \\
& \text { one or two }
\end{aligned}
$$

Deck 1-a consisted of 16 cards, 8 of which represented all possible cards which were positive instances of the concept "red and square", and 8 of which were randomly chosen from the remaining negative instances excluding "double negatives" such as blue circles. Deck l-b also contained 16 cards, 8 positive instances of the concept "red or square:" It was decided to use exclusive disjunction, so four of the positive instances were red circles, and four were blue squares, there being no red squares in the deck. The negative instances were all blue circles.

The second pair of decks was selected from the set of cards having the following attributes and values.

\section{Attribute}

card color

figure color

size of figure

shape of figure
Values

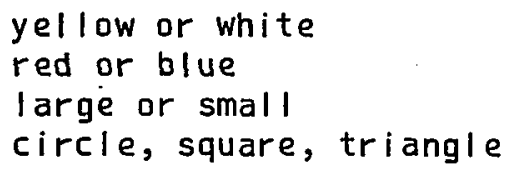

Each deck consisted of 12 cards. Deck 2-a was used for the conjunctive task where the correct concept was "large and white." six of the cards represented all possible positive instances in this set. The remaining six were drawn randomly from the negative instances with the stipulation that none of them be neither large nor white, that is, all were white and small or large and yellow. Deck 2-b consisted of 6 positive instances of the concept "large or white": three cards which were large, and three which were white, with no "double positives." The 6 negative 
instances were of course all small and yellow.

\section{Pracedure}

Each subject was tested individually in two sessions. Two tasks were performed in each session, one conjunctive and one disjunctive. Half of the subjects in each age group received the tasks in the following order: Session one--conjunctive, disjunctive; Session two-disjunctive, conjunctive. The other half had the order of the sessions reversed. Subjects were randomly assigned to the order they received, with the provision that the orders were evenly distributed between the sexes within each age group. The time interval between sessions varied from two hours to one day.

Prior to meeting any subjects, the correct concepts were chosen by the experimenter. The relevant attributes were chosen randomly from among"the possible attributes, as were the correct values. Each pair of decks was used for one conjunctive and one disjunctive task: deck $1-a$ for the first conjunctive task and deck $1-b$ for the second disjunctive task. Because there was no assurance that all the attributes would be equally attractive, and therefore equally easy for the subjectis, the same attributes and values were used for each pair of conjunctive and disjunctive tasks. Thus, the correct concept in conjunctive task one (deck 1-a) was "red and square." The correct concept in disjunctive task two (deck 1-b) was "red and square." The correct concepts when deck 2 was used were "white and large" and "white or large." The subjects were of course not told that the pairs would have this relationship. Nor were the subjects told that the number of 
positive and negative instances was equal.

Subjects who received a conjunctive task first were given the

following instructions, although the wording was somewhat modified for

the youngest and oldest groups.

Here are some cards for you to look at (cards are laid out on table in random order). There are five important things to notice about each card--five things that name a card and that make it different from all the other cards. They are: 1) the color of the card, which can be yellow or white (examples are pointed out for each value of each attribute); 2) the color of the figure, red or blue; 3) the shape of the figure, square or circle; 4) the size of the figure, large or small; 5) and the number of figures, one or two. Can you remember those five things? (They are practiced until the subject can name and give examples of the five attributes.)

The reason that it is important for you to remember those five things is that 1 'm going to use two of them in a rule for dividing all these cards into two groups. I'm going to make up a rule like this--all the cards that are both white and have two figures go in this group here (a positive instance is placed aside from the other cards) and all the cards that don't follow that rule go in another pile over here (place designated). Do you understand? Can you put the rest of the cards in the right groups? (Tasks of this kind are repeated until the subject demonstrates clear ability to complete them correctly.) Now l'm going to make up another rule, and this time l'm not going to tell you what it is because 1 want to see if you can figure it out. Do you understand? (Legitimate questions are answered.) I will give you one hint--this card (a positive instance is selected) follows the rule, so it goes in this group. Now you pick out other cards that you think follow the same rule, or pick out cards that you think don't follow the rule, and put them in the correct groups. 1'll tell you whether you're right or wrong. The most important thing is to try to place cards in the correct pile, but figuring out the rule will help you to do that.

The subject now selects a card and places it on one of the two piles. The experimenter asks why the subject chose that pile, records the response and the reason, and then tells the subject "right" or "wrong" If the response is wrong, the experimenter places the card in the cor- 
a

RESULTS

The principal measure taken was the number of errors committed during the task, that is, the number of cards originally placed in the incorrect group. The errors for the two disjunctive tasks were summed, as were the errors for the two conjunctive tasks, to obtain one score for each subject on each task. An analysis of varianc was performed on the scores. The results of this test are given in Table 1. It can be seen from Table I that the difference between the conjunctive and disjunctive tasks is significant, and that the age difference is significant. The lack of interaction between age and task indicates that the disjunctive task is harder at all ages, and thus fails to support the hypothesis that the disjunctive task becomes increasingly harder with age.

The magnitude of the differences can be seen in Figure 1 . It is quite striking that the differences in difficulty between conjunctive and disjunctive tasks is almost constant for all ages, except for the convergence at the youngest age group, which must be accepted as random variation.

A second measure, a reflection of the logic used in approaching the two tasks, is the appropriateness or inappropriateness of the initial choice of cards in each task by each subject. If the ideal initial choice is made in both conjunctive tasks and in neither disjunctive task, the subject is scored in the $C>D$ category. If the subject chooses the ideal initial card in the conjunctive task only once, and 
in the disjunctive task never, or if he chooses the ideal initial card in both conjunctive tasks and in only one disjunctive task, then he is placed in the $C>D$ category. If he performs equally well in both tasks, or if he chooses the ideal card more often in the disjunctive tasks, then he is placed in the $C \leq D$ category. The resulting distribution can be seen in Table 2. A chi ${ }^{2}$ performed on the data of Table 2 is significant at the .05 level. This indicates that the different age groups are differentially able to use the ideal approach to solving conjunctive and disjunctive problems. Specifically, the eight-and el even-year-olds perform much better on conjunctive tasks, while the five-, seven- and thirteen-year-olds perform only slightly better on conjunctive tasks. Another reflection of the logic used in approaching the tasks is whether the initial choice is a positive instance or a negative instance. The ideal choice for conjunctive tasks is positive, for disjunctive tasks is negative. The figures for both tasks are given in Table 3. Two independent $\mathrm{chi}^{2}$ tests were performed on these figures. The first, on the frequencies of positive and negative choices in conjunctive tasks, is nonsignificant. The second, for disjunctive tasks, is significant at the .0l level. This indicates that there are age differences for disjunctive but not for conjunctive tasks. All age groups choose primarily positive instances when solving conjunctive tasks, but only eight- and eleven-year-olds choose primarily positive instances when solving disjunctive tasks, the other groups choosing about half negative instances. 
TABLE ।

Analysis of variance

of ërror scores

\begin{tabular}{|c|c|c|c|c|c|}
\hline Source of variation & SS & $d f$ & MS & $F$ & \\
\hline \multicolumn{6}{|l|}{ Between subjects } \\
\hline Age & 332.98 & 4 & 83.25 & 10.30 & $p<.01$ \\
\hline $\operatorname{sex}$ & .37 & I & .37 & $-\infty$ & \\
\hline Age $x$ sex & 36.65 & 4 & $9: 16$ & $-\infty-$ & \\
\hline Subjects within & & & & & \\
\hline groups & 703.01 & 87 & & & \\
\hline \multicolumn{6}{|l|}{ Within subjects } \\
\hline $\begin{array}{l}\text { Task } \\
\text { Aqe } \mathrm{x} \text { task }\end{array}$ & $\begin{array}{l}79.26 \\
16.01\end{array}$ & $\begin{array}{l}1 \\
4\end{array}$ & $\begin{array}{r}79.26 \\
4.00\end{array}$ & 15.33 & $p<.01$ \\
\hline Sex $x$ task & .53 & $i$ & .53 & - - & \\
\hline $\begin{array}{l}\text { Age } X \text { sex } X \text { task } \\
\text { Task } X \text { subjects }\end{array}$ & 4.42 & 4 & 1.10 & - - & \\
\hline within groups & 449.78 & 87 & 5.17 & & \\
\hline
\end{tabular}


TABLE 2

Frequency of ideal response in conjunctive and disjunctive tasks.

\begin{tabular}{llllll} 
Age & 5 & 7 & 8 & 11 & 13 \\
\hline$C>D$ & 5 & 5 & 11 & 11 & 6 \\
$C>D$ & 7 & 6 & 7 & 7 & 3 \\
$C \leq D$ & 9 & 9 & 2 & 2 & 7
\end{tabular}

TABLE 3

Frequency of positive and negative responses in conjunctive and disjunctive tasks.

\begin{tabular}{crrrrr} 
Age & 5 & 7 & 8 & 11 & 13 \\
\hline Conjunctive task & $\ldots$ & & & & \\
pos (ideal) & 37 & 37 & 38 & 39 & 29 \\
neg & 5 & 3 & 2 & 1 & 3 \\
& & & & & \\
Dis junctive task & & & & & \\
pos (ideal) & 17 & 20 & 29 & 29 & 16 \\
neg (ideal & 25 & 20 & 11 & 11 & 16
\end{tabular}


FIGURE I

Number of errors as a function of age.

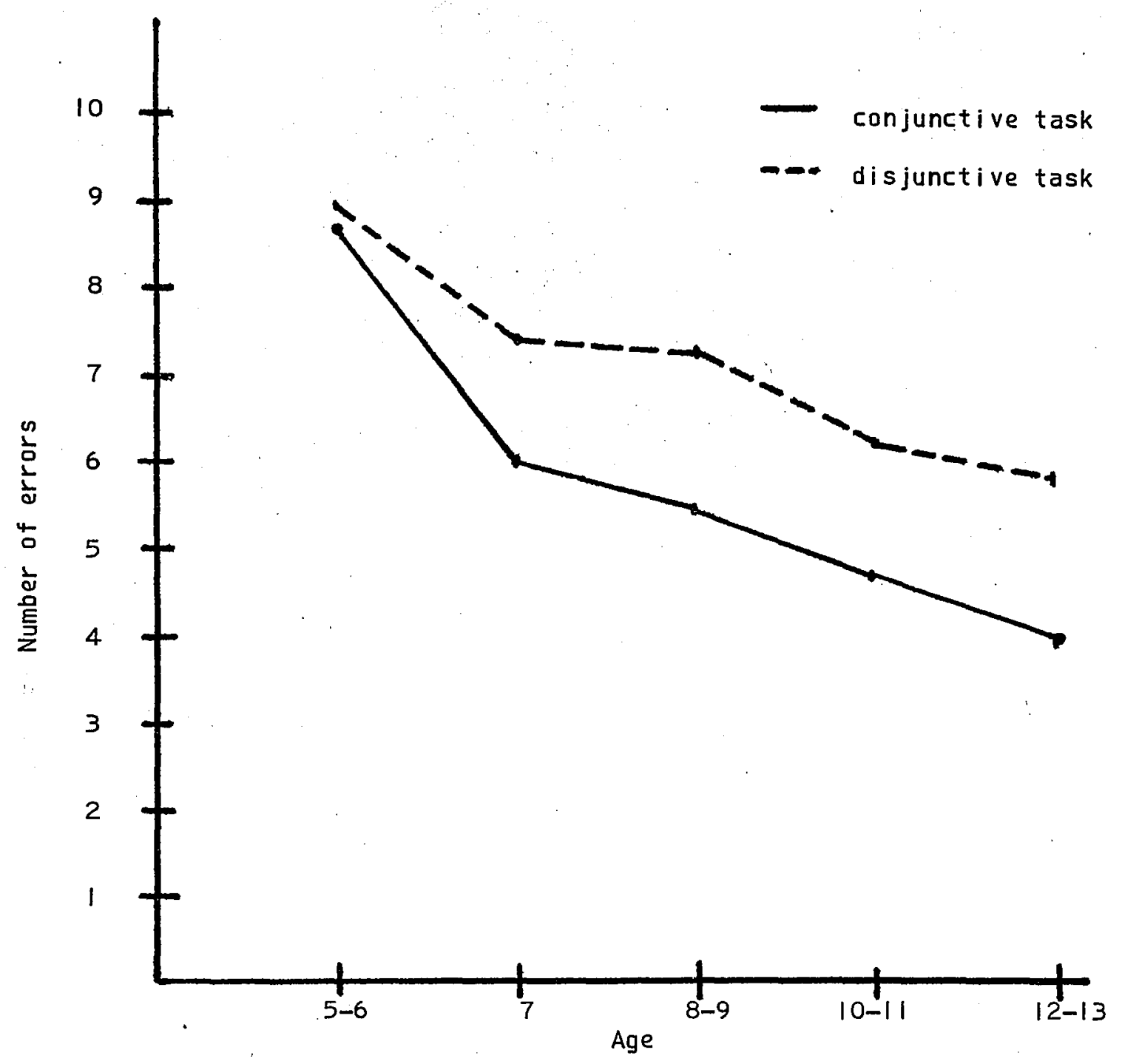


DISCUSSION

There'is little support for the hypothesis that disjunctive tasks are harder for adults simply because there is more opportunity to learn conjunctive reasoning. Either disjunctive tasks really are more difficult for children of all ages, or the youngest group tested in the present study has already been exposed to too many learning experiences where conjunctive reasoning was favored. It is difficult to explain why the curves in Figure I converge for the youngest age group, as the hypothesis suggested they should. This convergence might be a random event, but it suggests that perhaps more subjects at this lower age level should be tested. It might be even more instructive to test children who are slightly younger--perhaps four-yearolds, but practically speaking this would almost certainly require a new test. However, now that it is known that the absolute differences between the difficulty of conjunctive and disjunctive tasks is the same for children aged seven through thirteen, perhaps further experimentation could concentrate on three- through seven-year-olds, using simplified tasks which are appropriate for those ages. Another way of approaching the question of relative ability of children of different ages to attain disjunctive concepts would be to look at the strategies used at the various ages. Bruner in his book A Study of Thinking (1956) describes the kinds of strategies typically used by adults for conjunctive and disjunctive tasks. The striking feature is that adults used "positive focussing" strategies with dis- 
junctive tasks, even though thesejstrategies have no logical justification. There are two appropriate strategies for attaining disjunctive concepts; negative focussing, or finding a negative instance and changing one attribute at a time, is the most reliably efficient, but the multiple negative strategy, which is finding two or more negative instances, then taking the opposites of the values that are common to them, is aliso considered appropriate. Inappropriate but commonly used strategies all involve using positive instances as sources of information. The common element fallacy, fór instance, consists of noticing which values are common to all the positive instances and proposing them as the correct concept. Another version of the common element fallacy is the majority fallacy, which involves taking the elements common to most of the positive instances as the concept. Positive anchoring is an example of a strategy which wou!d be perfectly correct if applied to a conjunctive problem; it consists of finding two values in which a positive instance differs from a negative instance and proposing those as the concept: Majority anchoring means applying the strategy just described, but taking into account the majority effect, or the values common to the majority of the positive instances. Applying the same criteria as Bruner (1956) for appropriate strategies, do younger children differ from older ones in the correctness of their approach to the problem? Actually, there are some differences in procedure which must be taken into account. The subjects in this experiment were not being required to formulate hypotheses, so could not be expected to use precisely the same approaches. In the present study the subjects were selecting instances, and giving specific reasons 
why a given card belonged in a given group. It would be instructive to note whether they chose equally informative instances and gave equally cogent reasons in both kinds of tosks. In fact, it should be noticed that the tasks are precisely symmetrical; just as one can most efficiently attain a conjunctive concept by varying one attribute at a time, focussing on a positive instance, one can most efficiently attain a disjunctive concept by varying one attribute at a time, focussing on a negative instance, In both cases, because the relevant attributes are always two-valued, one is creating a conjunctive grouping in those instances where one is focussing, and a disjunctive grouping in the other instances. Are children of different ages equally capable of recognizing the symmetry. of the correct approaches to the two tasks? The answer to this question is suggested by the figures in Tables 2 and 3. Children of different aǵes do not perform equally well in choosing strategies for disjunctive tasks. A curvilinear relationship is suggested. The question arises of why, if there is an age difference in choice of strategies, there is no interaction of age with task when number of errors is used as the dependent measure, (Table 1). Perhaps younger children approach a disjunctive task with the advantage of not having learned the wrong strategy, but this advantage is offset by not being able to use the information available to them, so they make as many mistakes even though they have started off with an advantage. Or, perhaps the larger number of good disjunctive "strategies used by the five-year-olds (Tables 2 and 3 ) implies a more random performance at lower ages because the child is not yet at a stage of conceptual development which allows him to use a consistent 
approach. When he is able to use a more consistent approach he uses it with all the tasks given him. Since the first strategy available is conjunctive, he does well on conjunctive tasks but more poorly than before on disjunctive tasks. There would be more support for this last view if there were an improvement in conjunctive strategies simultaneous with the first appearance of difficulty with disjunctive strategies, at age 8 , but this is not the case. At any rate, there is no clear indication that the differential ability to apply disjunctive strategies is related to more opportunity to learn conjunctive strategies, so that the original facility with disjunctive tasks drops out. Perhaps, conjunctive strategies arise out of the random behavior of very. young children because they are inherently a more natural way of dealing with problems.

Taking into account that number of errors is a more reliable measure of ability than the nature of the first card chosen, the overall conclusion must be that disjunctive tasks are more difficult for children just as they are for aduits. The question of why this is true now presentsitself. Of course, any answer could be only speculation, but could lead to more specific attempts to answer the question. Bruner (1956) suggests one possibility when he points out that disjunctive categories violate the inductive principle. If a number of items are placed in one category, induction. would suggest that they are placed there for one reason. This is not possible in a disjunctive category where some of the items are there for one reason, and some for another. Is the inductive principle then a built in way of thinking? Or is it learned so early, because it is illustrated by every 
physical event, that all children think inductively before five years of age? Actually, studies with the Michotte phenomenon (0/um, 1956), comparing six- and seven-year-olds with adults, show that there are developmental differences in the perception of causality. Children perceive causality, less often than adults, indicating that the inductive principle is not a built-in way of thinking.

An intuitive assessment of the difference between conjunctive and disjunctive statements leads to the conclusion that disjunctive statements imply a lower level of certainty. From a conjunctive statement, such as "p $q$ " one can conclude "p" and one can conclude "q". In other words, one can make simple true statements as a direct conclusion to a conjunctive statement. From a disjunctive statement, such as " $p q$ ", however, one can conclude neither "p" nor "q" with certainty, until some other proposition is also stated. In terms of a concept formation task, one can conclude from knowing that a given card is a positive instance of the concept "red and square" that it is red and that it is square, knowing that it is a positive instance of "red or square" tells us nothing about any individual card until we also find out, for instance, that it is "not red" in which case we can conclude that it is square. Perhaps disjunctive concepts are more difficult because people have learned to demand a higher level of certainty or predictability than they allow. It is somehow undesirable or unsatisfactory to create a grouping of cards which are "red or square" because creating that group increases one's knowledge about the cards in it so little. Because disjunctive groups offer a level of predictability lower than is satisfactory, certainly lower than 
is normally possible when dealing with physical objects, people just do not spontaneously think in terms of such groups, and find them difficult to reason about logically.

The possibility must be considered that the brain is organized so that conjunctive solutions are easier. Perhaps a conjunctive idea is somehow simpler in terms of brain processes. This would mean that "red and square" is encoded in a simpler or more unitary way than "red or square". It might be possible to test this hypothesis by measuring the amount of time it takes subjects to form conjunctive versus disjunctive groupings when they know the concept. If "red and square" is an intrinsically simpler idea, then one would predict that less time would be required to pick out all positive instances of that concept from a large array of cards than to pick out all positive instances of the equivalent disjunctive concept. Possibly looking for "red or square" requires; two discrete steps in the thought process, whereas looking for "red and square" requires only one. If this is the case, then conjunctive reasoning would be more natural and easier for all ages, and probably conjunctive groupings would be more common.

The results of the present experiment lead to the conclusion that there is some aspect of disjunctive groupings which is inherently less economical than conjunctive groupings. As a result, conjunctive groupings are preferred and probably occur more frequently, resulting in more opportunities to reason about them and making it more useful to be able to reason about them correctly. Disjunctive strategies themselves are probably not inherently more difficult, but they are less practiced because they are rarely needed. 
SUMMARY

In light of the finding. (Bruner, 1956) that disjunctive concepts are more difficult for adults than conjunctive concepts, a developmental study of the relative difficulty of conjunctive and disjunctive concepts was undertaken. Five groups of children, ranging. in age from five years to thirteen years, were tested on logically equivalent conjunctive and disjunctive concepts. It was found that children of all ages make more errors on disjunctive than on conjunctive tasks. The strategies used for conjunctive tasks were more appropriate at all age levels. Because of the fact that the difference in difficulty is quite constant over the age groups, it was concluded that some aspect of conjunctive groupings is more natural. As a result, they occur more frequently and there is greater opportunity to learn to reason correctly about them. 
REFERENCES

Bruner, J.S., Goodnow, J.J. and Austin, G.A. A study of thinking. New York: John. Wiley \& Sons, 1956.

Bruner, J.S., Olver, R.R., Greenfield, P.R. et al. Studies in cognitive growth. New York: John Wil ley \& Sons, 1966.

Hovland, C.L. and. Weiss, W. Transmission of information concerning concepts through positive and negative instances. Journal of Experimental Psychology, $1953, \underline{45}, 175-182$.

Inhelder, B. and Piaget, J. The growth of logical thinking from childhood to adolescence. New York: Basic Books, 1964.

Olum, Vivian. Developmental differences in the perception of causality. American Journal of Psychol ouy, 1956, 69, 417-423.

Piaget, J. The construction of reality in the child. New York: Basic Books, 1954. 